\title{
Photocatalytic ammonia production enhanced by a plasmonic near-field and hot electrons originating from aluminium nanostructures $\uparrow$
}

\author{
Madasamy Thangamuthu, (D) * Christian Santschi \\ and Olivier J. F. Martin (D)
}

Received 4th October 2018, Accepted 20th November 2018

DOI: $10.1039 / c 8 f d 00146 d$

\begin{abstract}
Ammonia production at room temperature and atmospheric pressure is in high demand to assist in energy saving and the protection of the environment worldwide, as well as to help reduce $\mathrm{CO}_{2}$ emissions. Recently, plasmonic nanomaterials have been frequently used for solar to chemical energy conversion, which has the potential to replace existing energyintensive industrial processes. In our approach, plasmonic aluminium nanotriangles (AINTs) were used to investigate the impact of plasmonic effects on photocatalytic ammonia production. Plasmonic near-field coupling to a semiconductor and hot electron generation from AINTs were studied in detail through the use of electrochemical photocurrent measurements. A narrowband LED beam with a central wavelength at $365 \mathrm{~nm}$ was used to illuminate the AINTs and their hot electron generation efficiency was estimated to be $2 \times$ $10^{-4} \%$, resulting in an ammonia production rate of $4 \times 10^{-5} \mu \mathrm{M} \mathrm{h}^{-1} \mathrm{~mW}^{-1} \mathrm{~cm}^{-2}$, which corresponds to a quantum efficiency of $2.5 \times 10^{-5} \%$. In the case of plasmonic near-field coupling, AlNTs-embedded $\mathrm{TiO}_{2}$ demonstrates a charge-carrier generation efficiency of $2.7 \%$, which is $\sim 2.3$ times higher than that of bare $\mathrm{TiO}_{2}$. The ammonia production rate of AINTs- $\mathrm{TiO}_{2}$ is $0.1 \mu \mathrm{M} \mathrm{h}^{-1} \mathrm{~mW}^{-1} \mathrm{~cm}^{-2}$ with a quantum efficiency of $\sim 0.06 \%$, which corresponds to $\sim 2.4$ times that of the rate demonstrated by bare $\mathrm{TiO}_{2}\left(0.04 \mu \mathrm{M} \mathrm{h}^{-1} \mathrm{~mW}^{-1}\right.$ $\mathrm{cm}^{-2}$, quantum efficiency $\sim 0.025 \%$ ). The obtained results confirm successful ammonia production through nitrogen splitting at room temperature and under atmospheric pressure. Moreover, according to the presented results, the use of plasmonic aluminium structures remarkably improves the ammonia production rate.
\end{abstract}

\section{Introduction}

After photosynthesis, nitrogen fixation is the second most important chemical process in the biosphere, as nitrogen is an essential component of the building 
blocks of life, viz. DNA, RNA and proteins. Unfortunately, direct appropriation from its abundance in the atmosphere (78\%) is impossible since the robust triple bond between two nitrogen atoms makes molecular nitrogen relatively inert. The rhizobia bacteria of the legume family and which live in nodules on plant roots possess a nitrogenase enzyme which fixes nitrogen naturally and supplies it to plants and animals in the form of ammonium, urea and nitrate. ${ }^{1}$ This naturally occurring process is, however, not sufficient to feed the rapidly expanding population of Earth. For decades the agro-food industry has relied on nitrate fertilizers, produced via the Haber-Bosch industrial synthesis of ammonia $\left(\mathrm{NH}_{3}\right)$, to boost food production. In principle, $\mathrm{NH}_{3}$ can be produced in the presence of catalysts at high temperature $\left(400-600{ }^{\circ} \mathrm{C}\right)$ and pressure (150-200 atm), contributing to approximately $2 \%$ of the world's annual energy usage. ${ }^{2}$ Furthermore, industrial $\mathrm{NH}_{3}$ production consumes approximately $5 \%$ of the world's natural gas resources to produce a hydrogen feedstock, and releases large quantities of carbon dioxide $\left(\mathrm{CO}_{2}\right)$ which strongly pollutes the atmosphere, thus leading to serious environmental issues, viz. global warming and climate change etc. ${ }^{3}$ Therefore, there is strong demand for the development of alternative, fossil-fuelsaving methods that can be utilized to produce ammonia under ambient conditions in order to save energy and the environment.

Numerous researchers have attempted to identify an alternative method to the Haber-Bosch process via the use of an electrochemical approach. ${ }^{4}$ Recently, Licht et al. introduced an electrolysis method in which a sodium and potassium hydroxide solution mixture is bubbled in wet air through a mixture of tiny particles of iron oxide $\left(\mathrm{Fe}_{2} \mathrm{O}_{3}\right){ }^{5}$ This method consumes two-thirds of the energy consumed by the Haber-Bosch process and, even more appealingly, $\mathrm{CO}_{2}$ emissions are significantly reduced in comparison. Although electrochemical routes reduce energy input, they are still under development due to numerous technical challenges and material issues. In another approach, inorganic metal complexes were studied to mimic nitrogenase activity for nitrogen adsorption and reduction. ${ }^{6,7}$ However, this route is limited because of the complicated synthesis of the materials and their poor stability. Semiconductor photocatalysis for ammonia production $^{8-10}$ has also been highly studied for a few decades after the Fujishima and Honda water splitting experiments were reported..$^{11}$ To improve the quantum efficiency and reduce charge-carrier recombination, Khan et al. and Ranjit et al. studied transition-metal-loaded semiconductors. ${ }^{12-15}$ Unfortunately, the efficiency was not significantly improved.

Recently, plasmonic nanomaterials (PNs), a new family of catalysts exhibiting fundamentally different behaviour, have been shown to be highly encouraging due to their optical and catalytic properties under visible light illumination at low intensity. The photocatalytic efficiency can be greatly enhanced by combining PNs with a semiconductor, which increases charge-carrier generation in the semiconductor through plasmonic near-field coupling. ${ }^{16}$ Moreover, PNs can solely generate charge-carriers (electron-hole pairs) through non-radiative surface plasmon decay. ${ }^{17}$ These so-called plasmonic hot electron/hot hole pairs (EHP) have the potential to drive chemical reactions either on the PNs or on the semiconductor surface, where plasmonic hot electrons can be extracted and transferred to the conduction band of the semiconductor. A Schottky barrier at the metal/semiconductor junction prolongates the residence time of the electrons in the conduction band and, therefore, reduces the fast recombination rate of the 
short living EHP. ${ }^{18}$ So far, such a plasmonic approach has been successfully applied to improve water splitting, ${ }^{19}$ hydrogen dissociation, ${ }^{20,21}$ the decomposition of organic pollutants such as carbon dioxide, ${ }^{22}$ carbon monoxide ${ }^{23}$ and dyes, ${ }^{24}$ and the synthesis of organic compounds. ${ }^{25,26}$ Recently, Oshikiri et al. studied, using light in the visible range, hot electron generation from $\mathrm{Au}$ nanoparticles combined with a ruthenium co-catalyst for ammonia synthesis, and encountered a poor yield. ${ }^{27}$ In a follow-up study they used zirconium/zirconium oxide, an efficient nitrogen adsorbing material that improved ammonia production significantly. ${ }^{28}$ However, $\mathrm{Au}$ and $\mathrm{Ag}$ are not seminal materials since they are very expensive and unstable over long periods of time, therefore making them inappropriate for long-term use and, consequently, commercialization. Furthermore, the thermal stabilities of $\mathrm{Au}$ and $\mathrm{Ag}$ are inferior to the stability of $\mathrm{Al}$, therefore rendering $\mathrm{Au}$ and $\mathrm{Ag}$ unsuitable for high temperature annealing.

In our work we used aluminium as a plasmonic material. Aluminium is a cheap material that exhibits plasmonic properties in the UV range, and is, in comparison with other plasmonic metals, more abundant on earth, highly stable and non-toxic. To the best of our knowledge, we are the first group to study the use of aluminium nanotriangles (AlNTs) for enhancing ammonia production through nitrogen splitting at room temperature and under atmospheric pressure. In order to gain more insight into the plasmonic mechanisms, two different types of photoelectrodes were fabricated to study: (i) plasmonic hot electron generation from AlNTs and (ii) plasmonic near-field coupling to semiconductor materials, such as $\mathrm{TiO}_{2}$. The charge-carrier generation rate of the photoelectrodes under $365 \mathrm{~nm}$ illumination was studied via electrochemical photocurrent measurements. An incident photon to photocurrent generation efficiency (IPCE) of $2 \times$ $10^{-4} \%$ was observed for the hot electron generation by non-radiative decay of surface plasmons, resulting in an ammonia production rate of $4 \times 10^{-5} \mu \mathrm{M} \mathrm{h}^{-1}$ $\mathrm{mW}^{-1} \mathrm{~cm}^{-2}$, which corresponds to a quantum efficiency of $2.5 \times 10^{-5} \%$. However, after applying plasmonic near-field coupling, the IPCE increases to $2.7 \%$, which is $\sim 2.3$ times higher than that observed when using a bare $\mathrm{TiO}_{2}$

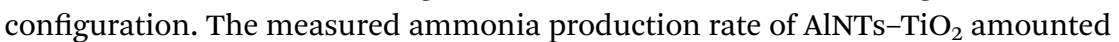
to $0.1 \mu \mathrm{M} \mathrm{h}^{-1} \mathrm{~mW}^{-1} \mathrm{~cm}^{-2}$ with a quantum efficiency of $\sim 0.06 \%$, which is $\sim 2.4$ times higher than that obtained with bare $\mathrm{TiO}_{2}\left(0.04 \mu \mathrm{M} \mathrm{h}^{-1}\right.$, quantum efficiency $\sim 0.025 \%$ ).

\section{Experimental}

\subsection{Preparation of the photoelectrodes}

Plasmonic aluminium nanotriangles (AlNTs) with a side length of $85 \mathrm{~nm}$ were fabricated on fused silica substrates via colloidal lithography (see ESI $\dagger$ for the detailed fabrication procedure). Due to the high reactivity of Al with oxygen, a selflimiting oxide layer formed on the $\mathrm{Al} /$ air interface. Al was deposited via electron beam (e-beam) evaporation with a base pressure between $10^{-6}$ and $10^{-7}$ Torr

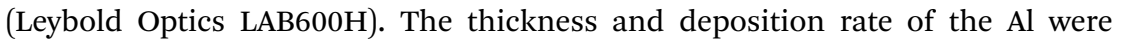
$40 \mathrm{~nm}$ and $0.5 \AA \mathrm{s}^{-1}$, respectively. ${ }^{29}$ Subsequently, the PS mask was removed by sonication in toluene for $10 \mathrm{~min}$, followed by the deposition of a $100 \mathrm{~nm}$ thick titanium dioxide $\left(\mathrm{TiO}_{2}\right)$ thin film onto the AlNTs via the e-beam evaporation technique with a deposition rate of $1 \AA^{-1} \mathrm{~s}^{-1}$ prior to an annealing step at $500{ }^{\circ} \mathrm{C}$ for 


\section{(A)}

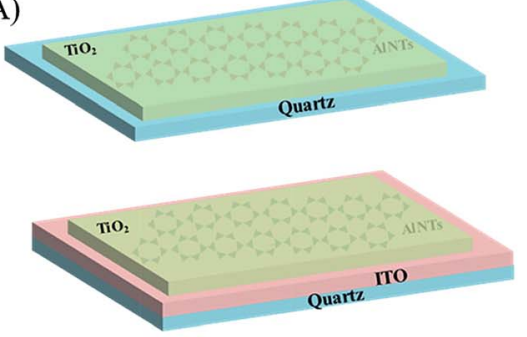

(C)

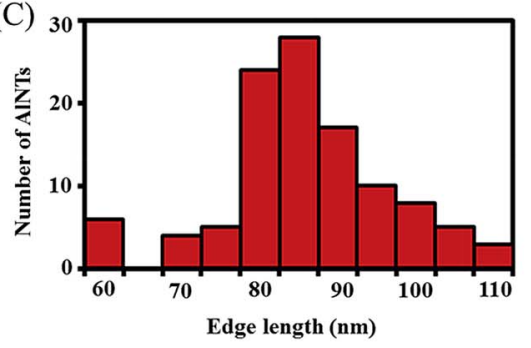

(B)

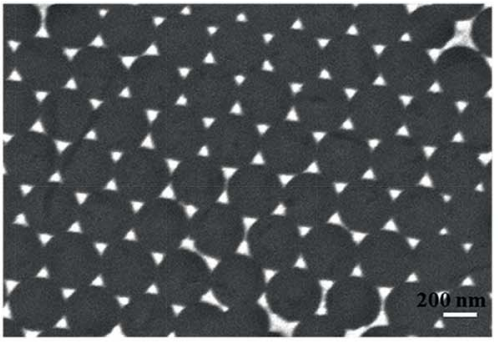

(D)

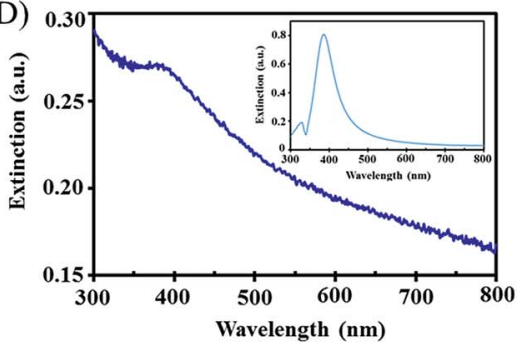

(E)

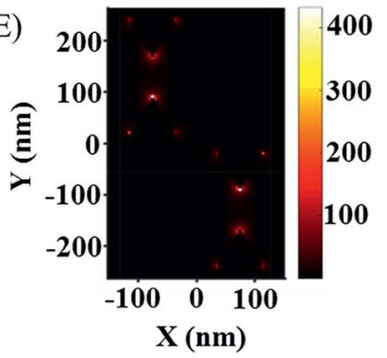

Fig. 1 Characterization of the photoelectrodes. (A) Schematics of the photoelectrodes. (B) SEM image of the AINTs. (C) Particle size distribution of the AINTs. (D) UV-Vis-NIR extinction spectrum and the simulated optical spectrum of the AINTs (inset). (E) Simulated spatial distributions of the electric field enhancement for the AINTs.

$2 \mathrm{~h}$ under a nitrogen atmosphere. Fig. 1A shows schematics of the photoelectrodes.

\subsection{Characterization}

A UV-Vis-NIR spectrometer (UV-2600) with an integrating sphere was used for the extinction, absorption and scattering measurements. The electrical field distribution around the excited AlNTs was computed using the surface integral equation (SIE) method. ${ }^{30}$ The real dimension, obtained from the scanning electron microscope (SEM) images, was introduced to calculate the optical responses of the AlNTs. All dielectric coefficients were taken from the literature. ${ }^{31-33}$ Morphological SEM images were obtained using a Zeiss Merlin field emission scanning electron microscope (Carl Zeiss Jena $\mathrm{GmbH}$ ) with an electron energy of $2 \mathrm{keV}$. Xray diffraction (XRD) characterization was carried out using an Empyrean diffractometer from Panalytical equipped with a grazing incidence angle configuration and $\mathrm{Cu} K \alpha$ radiation source $(\lambda=0.15418 \mathrm{~nm})$ in order to study the 
crystalline structure of the $\mathrm{TiO}_{2}$. The bandgap energy of the fabricated $\mathrm{TiO}_{2}$ was calculated using a Tauc-plot. The valence band spectrum of the $\mathrm{TiO}_{2}$ was obtained using a PHI versaprobe II scanning XPS microscope (Physical Instruments AG, Germany). The analysis was carried out using a monochromatic Al $\mathrm{K} \alpha \mathrm{X}$-ray source of $24.8 \mathrm{~W}$ with a beam size of $100 \mu \mathrm{m}$, and the binding energy was calibrated using a value for C 1 s of $187.85 \mathrm{eV}$.

\subsection{Electrochemical photocurrent study}

To perform the electrochemical studies, an electrical conducting layer was added between the substrate and the AlNTs or $\mathrm{TiO}_{2}$. A $150 \mathrm{~nm}$ ITO thin film was deposited via radio frequency magnetron sputtering (RF-MS, Pfeiffer SPIDER 600) with an RF power of $500 \mathrm{~W}$ and an argon flow of $15 \mathrm{sccm}$ at $5 \times 10^{-3} \mathrm{mbar}$. The AlNTs embedded in $\mathrm{TiO}_{2}$ photoelectrodes on ITO were fabricated as described above. Electrochemical measurements were performed using a CHI 1240B potentiostat ( $\mathrm{CH}$ Instruments, Austin, USA). A three electrode photoelectrochemical cell (Zahner instruments) consisting of a photoanode (working electrode), a $\mathrm{Ag} / \mathrm{AgCl}$ reference electrode and a platinum counter electrode was used to record the photocurrent. A $0.1 \mathrm{M} \mathrm{KNO}_{3}(\mathrm{pH} \sim 6)$ solution containing $10 \%$ ethanol as an electron donor was used as the electrolyte. The working electrode potential was set to $0.15 \mathrm{~V} v s$. $\mathrm{Ag} / \mathrm{AgCl}$ for all of the experiments.

\subsection{Photocatalysis experiments}

A photochemical cell was used as the reaction chamber. A collimated narrowband LED beam with its central wavelength at $365 \mathrm{~nm}$ was used as the irradiation source. The fabricated photoelectrodes were illuminated with an intensity of $5 \mathrm{~mW} \mathrm{~cm}^{-2}$ on a circular area with a diameter of $1.5 \mathrm{~cm}$. Unless otherwise stated, the illumination conditions were the same for all measurements. A nitrogen-saturated aqueous electrolyte solution ( $\mathrm{pH}$ 4) containing 10\% ethanol, an electron donor/hole scavenger, was used for the reaction. The solution contained $\sim 500 \mu \mathrm{M}$ (saturated concentration) of nitrogen, corresponding to $3 \times 10^{16}$ nitrogen molecules in the reaction chamber. This concentration was held constant throughout the whole experiment by using a $100 \mathrm{sccm}$ nitrogen flow. The nitrogen gas flow rates were controlled using a mass flow controller (Omega, GmbH). The rate of ammonia production was evaluated by a standard colorimetric assay using sodium salicylate, sodium hypochlorite, sodium nitroprusside and sodium hydroxide. A $500 \mu \mathrm{L}$ sample was taken from the reaction chamber at $1 \mathrm{~h}$ intervals. Subsequently, $250 \mu \mathrm{L}$ of hypochlorite solution followed by $250 \mu \mathrm{L}$ of salicylate reagent (a mixture of sodium salicylate, nitroprusside and sodium hydroxide) were added. Sodium salicylate forms a dimer (indosalicylate) in the presence of ammonia, with an absorption peak at $650 \mathrm{~nm}$. Known amounts of ammonium chloride were used to perform a calibration. The value of the ammonia concentration at $t=0 \mathrm{~h}$ was used as the baseline and subtracted from all measurements. The quantum efficiency of ammonia formation for which three photons are required to produce one ammonia molecule was determined using the following eqn $(1)^{34}$

$$
\eta_{\mathrm{NH}_{3}}(\%)=\frac{\left[\text { Rate of } \mathrm{NH}_{3} \text { formation }\left(\mathrm{mol} \mathrm{s}^{-1}\right)\right] \times 3}{\left[\text { Incident photon flux }\left(\mathrm{mol} \mathrm{s}^{-1}\right)\right]} \times 100
$$




\section{Results and discussion}

\subsection{Photoelectrodes characterization}

An SEM image of the AlNTs is shown in Fig. 1B. Using Image J, the size distribution of the AlNTs was statistically evaluated, as shown in Fig. 1C which reveals that the average edge length of the AlNTs is approximately $85 \mathrm{~nm}$, leading to a surface coverage of $\sim 5 \%$. The UV-Vis-NIR extinction spectrum shows a dipolar plasmon peak at around $365 \mathrm{~nm}$ (Fig. 1D). The position of the plasmonic resonance wavelength matches well with the simulated spectrum (inset of Fig. 1D).

Furthermore, the SIE near-field simulations show that the highest plasmonic electric field intensities at the resonance position are generated at the edges of the AlNTs (Fig. 1E). The crystalline nature of the $\mathrm{TiO}_{2}$ was studied before and after annealing using XRD. Fig. 2A displays the XRD pattern for $\mathrm{TiO}_{2}$ on ITO before annealing. The crystalline peak for anatase $\mathrm{TiO}_{2}$ is completely absent, indicating that the as-deposited $\mathrm{TiO}_{2}$ is amorphous. The peaks observed at the $2 \theta$ angles of $30.4,33,35.34$ and $37.65^{\circ}$ correspond to the (222), (312), (004) and (114) polycrystalline phases, respectively, of the ITO (JCPDS: 96-101-0342) underneath. However, the XRD pattern of the annealed sample (Fig. 2B) shows an obvious peak at $25.3^{\circ}$, thus confirming the anatase phase (101) (JCPDS: 00-021-1272). A reorganization of the $\mathrm{TiO}_{2}$ morphology is also evident from analysis of the SEM images (insets of Fig. 2A and B), in which a clear change in the crystal size can be observed. The optical band gaps $\left(E_{\mathrm{g}}\right)$ of $\mathrm{TiO}_{2}$ on the fused silica and ITO substrates were calculated using Tauc plots, as shown in Fig. 2C. The quantity $(\alpha h \nu)^{1 / 2}$ was plotted as a function of the incident energy and, subsequently, the linear part was extrapolated (see ESI $\uparrow$ for details). The resulting point of intersection with the abscissa at $3.18 \mathrm{eV}$ corresponds to the bandgap energy of the deposited $\mathrm{TiO}_{2}$. The measured bandgap value is in good agreement with the literature..$^{35}$ The valence band (VB) spectrum of the $\mathrm{TiO}_{2}$ was determined via XPS analysis (Fig. 2D) in order to obtain the potential at the upper edge of the VB. ${ }^{35}$ The VB spectrum shows that the valence band is located at $2.7 \mathrm{eV}$ below the Fermi level, and hence the lower edge of the CB can be expected to be located at $0.48 \mathrm{eV}$ above the Fermi level.

The lower conduction band edge was experimentally determined using photocurrent onset measurements, ${ }^{36}$ as shown in Fig. 2E. Under illumination by light at $365 \mathrm{~nm}$ (an energy higher than the band gap), $\mathrm{TiO}_{2}$ generates EHP, which are separated in the potential gradient space-charge layer (formed upon immersing the electrode in the electrolyte). This drives holes towards the $\mathrm{TiO}_{2} /$ electrolyte interface and electrons towards the bulk of the $\mathrm{TiO}_{2}$ as well as to an external electrical connection (Fig. 2F). Consequently, an anodic photocurrent is observed in the $\mathrm{TiO}_{2}$ photoelectrode which is affected by an external bias. The potential of the electrode swept in the cathodic direction (applying a more negative potential) reduces the space-charge layer thickness, thus resulting in more charge-carrier recombination and leading to a decreased photocurrent. The photocurrent becomes zero at the flatband potential. In Fig. 2E, the photocurrent reaches zero at $-0.1 \mathrm{~V}$ with respect to $\mathrm{Ag} / \mathrm{AgCl}$, which is equal to $-0.335 \mathrm{~V} v s$. a standard hydrogen electrode (SHE). For $\mathrm{TiO}_{2}$, recombination is practically complete $0.2 \mathrm{~V}$ away from the $\mathrm{CB}$ edge, therefore this value is added to the flatband potential in order to calculate the final CB edge value. ${ }^{37}$ Hence, the CB edge of the $\mathrm{TiO}_{2}$ should be located at $-0.54 \mathrm{~V}$ above the Fermi level, which is very close 
(A)

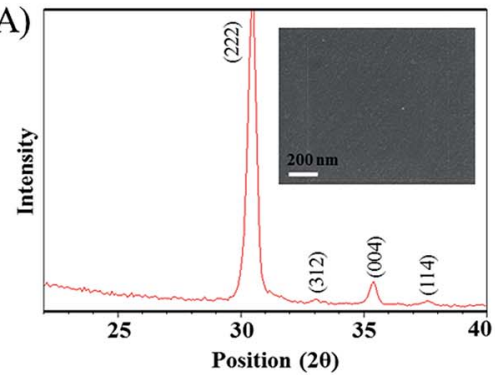

(C)

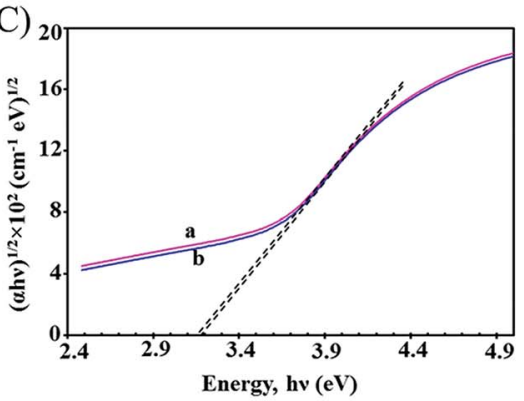

(E)

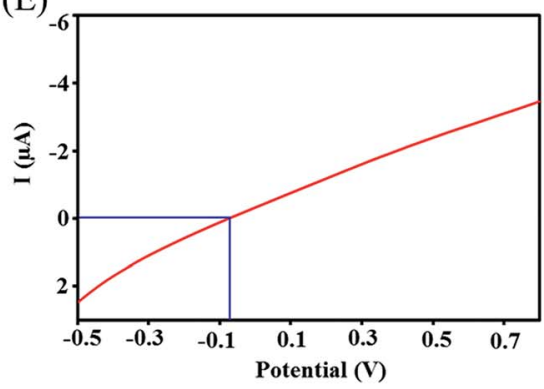

(B)

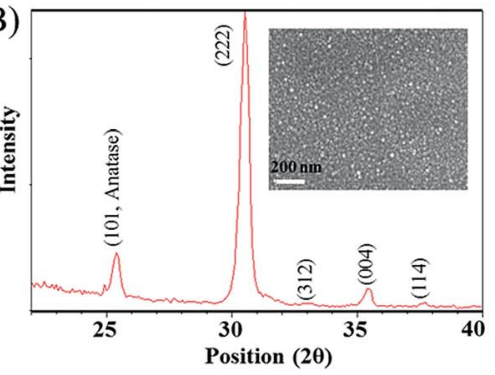

(D)

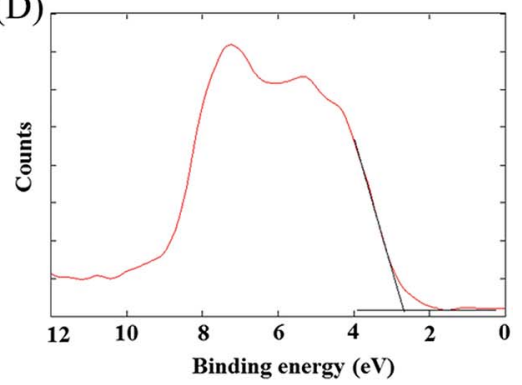

(F)

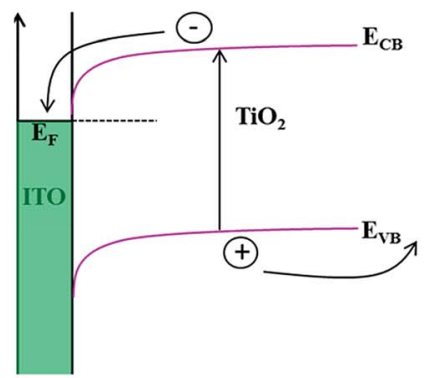

Fig. 2 XRD patterns of the $\mathrm{TiO}_{2}$ (A) before annealing and (B) after annealing at $500{ }^{\circ} \mathrm{C}$ for $2 \mathrm{~h}$ under a nitrogen atmosphere (the insets show SEM images of the $\mathrm{TiO}_{2}$ before and after annealing). (C) Tauc-plot of $(\alpha h \nu)^{1 / 2}$ as a function of the incident photon energy $(h \nu)$ for $\mathrm{TiO}_{2}$ on the fused silica (a) and ITO (b) substrates. (D) Valence band photoelectron spectrum of $\mathrm{TiO}_{2}$ (anatase) after annealing. (E) Photovoltammogram of the $\mathrm{ITO}-\mathrm{TiO}_{2}$ electrode recorded in a $\mathrm{KNO}_{3}(0.1 \mathrm{M} ; \mathrm{pH}$ 6) electrolyte in the cathodic direction with a sweep rate of $2 \mathrm{mV} \mathrm{s}^{-1}$ under $365 \mathrm{~nm}$ irradiation. (F) Schematic showing the $\mathrm{TiO}_{2}$ energy levels under illumination.

to the $\mathrm{CB}$ edge value deduced from the Tauc-plot. The band diagram of the $\mathrm{TiO}_{2}$ is depicted in Fig. 3A.

\subsection{Electrochemical study of the plasmonic effects of the AlNTs}

The plasmonic effects of the AlNTs were studied by means of electrochemical photocurrent measurements. The number of charge-carriers generated in the photoelectrodes is estimated to correspond to a direct measure of their photocatalytic activity. ${ }^{38}$ In the first step, we studied plasmonic hot electron generation from the AlNTs. Fig. 3B shows the photocurrent responses of the ITO-AlNTs and 
(A)

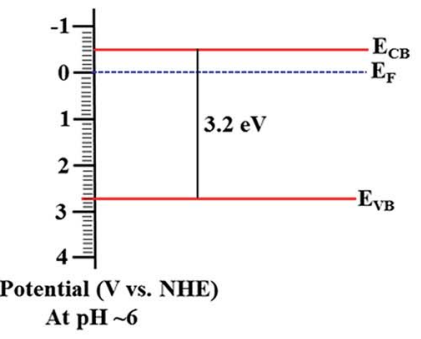

(C)

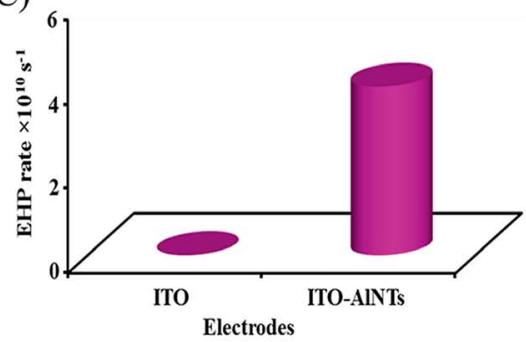

(B)

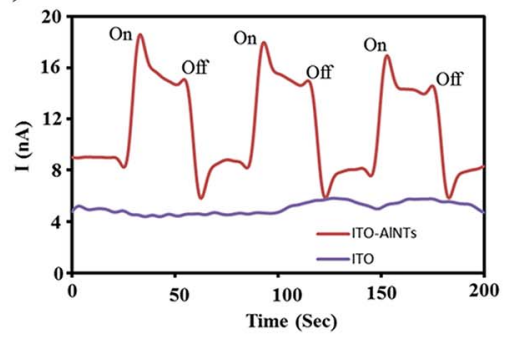

(D)

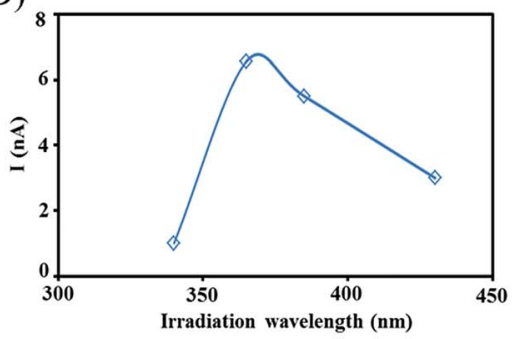

(E)

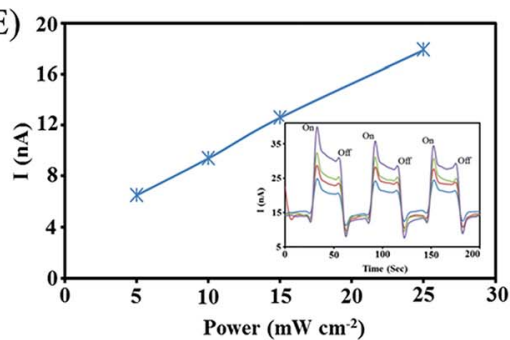

Fig. 3 (A) Energy band diagram of anatase $\mathrm{TiO}_{2}$ on the electrochemical potential scale; $\mathrm{NHE}$ refers to the normal hydrogen electrode. (B) Photocurrent of bare ITO and ITOAINTs in a $0.1 \mathrm{M} \mathrm{KNO}_{3}(\mathrm{pH} \sim 6)$ solution containing $10 \%$ ethanol, recorded under narrowband irradiation at $\lambda=365 \mathrm{~nm}\left(5 \mathrm{~mW} \mathrm{~cm}^{-2}\right)$. (C) Charge-carrier generation rate of the photoelectrodes with and without AINTs. (D) Wavelength dependent photocurrent of the ITO-AINTs photoelectrode observed using 340, 365, 385 and $430 \mathrm{~nm}$ narrowband light sources at $5 \mathrm{~mW} \mathrm{~cm} \mathrm{~cm}^{-2}$ power. (E) Observed photocurrent as a function of light intensity. The inset shows the photocurrent responses of the ITO-AINTs electrode at $365 \mathrm{~nm}$ under different light intensities (from bottom to top: 5, 10, 15 and $25 \mathrm{~mW} \mathrm{~cm}^{-2}$ ). The electrodes were biased with $0.15 \mathrm{~V}$ for all of the photocurrent measurements.

bare ITO samples. The $365 \mathrm{~nm}$ light source was switched on and off every $30 \mathrm{~s}$ whilst the photocurrent response was recorded. The obtained currents were stable and reproducible. The ITO-AlNTs sample shows significant photocurrent changes of $\Delta I=6.5 \mathrm{nA}$ under illumination, which corresponds to a generation rate of $4 \times 10^{10}$ hot electrons/holes per second (Fig. 3C) and an incident photon to photocurrent conversion efficiency (IPCE), i.e. external quantum efficiency, of $2 \times$ $10^{-4} \%$. This result clearly suggests that the AlNTs generate hot electrons as a result of non-radiative surface plasmon decay. The bare ITO does not demonstrate a significant change in current, thus indicating that the illumination wavelength of $365 \mathrm{~nm}$ does not excite the ITO. Furthermore the bandgap of ITO is large, around $4 \mathrm{eV}$, meaning that it certainly requires a higher energy $(300 \mathrm{~nm}$ 
wavelength) to generate charge-carriers; this confirms that the observed $6.5 \mathrm{nA}$ photocurrent is only due to the plasmonic hot electrons from the AlNTs. The wavelength dependent hot electron generation efficiency was also studied using different illumination wavelengths, as shown in Fig. 3D. The maximum current was observed at $365 \mathrm{~nm}$, thus providing further evidence of hot electron generation. To gain more insight into the plasmonic response of the AlNTs, the photocurrent responses at different light intensities were studied. It is clear from looking at Fig. 3E that the AlNTs are linearly responding to the light intensity, which is a characteristic of plasmonic nanomaterials. ${ }^{39}$

In the second step, plasmonic near-field coupling was studied by connecting the AlNTs to $\mathrm{TiO}_{2}$. Fig. 4A shows the photocurrent responses of the ITO-TiO samples with and without AlNTs under dark and illuminated conditions. For the bare $\mathrm{TiO}_{2}$ electrode, a photocurrent of $I=33.2 \mu \mathrm{A}$ was observed, corresponding to a generation rate of $2.1 \times 10^{14}$ EHP per second (Fig. 4B) and an IPCE of 1.1\%. The photocurrent response increased to $I=78 \mu \mathrm{A}$ for the sample with AlNTs, corresponding to a generation rate of $4.9 \times 10^{14}$ EHP per second and an IPCE of $2.7 \%$. Overall, a $\sim 2.3$ fold enhancement was observed after embedding the AlNTs into $\mathrm{TiO}_{2}$, which is also manifested in the increase in the absorption spectrum, as shown in Fig. 4C. The wavelength dependent photocurrent enhancement of the ITO-AlNTs- $\mathrm{TiO}_{2}$ electrode was determined and is shown in Fig. 4D, which reveals that the enhancement strongly depends on the illumination wavelength, which qualitatively follows the absorption spectrum of the AlNTs (inset of Fig. 4D). The peak position of the photocurrent enhancement and the AlNTs absorption matches very well, thus suggesting that the electromagnetic field located at the edges of the triangles, generated by the dipolar surface plasmon oscillation of the AlNTs, penetrates into the $\mathrm{TiO}_{2}$, leading to an increased charge-carrier generation rate.

It is worth noting that the AlNTs were in direct contact with $\mathrm{TiO}_{2}$. The $\mathrm{Al}$ and $\mathrm{TiO}_{2}$ contact exhibits ohmic character since the working function of $\mathrm{Al}$ is inferior to that of $\mathrm{TiO}_{2}$. Therefore, plasmonic hot electron and near-field coupling effects are indistinguishable in the studied configuration. However, the observed results suggest that the hot electron generation and extraction efficiency is very low $(2 \times$ $10^{-4} \%$ ) in comparison with the charge-carrier generation efficiency of the nearfield coupling $(2.7 \%)$, thus demonstrating that the contribution from the hot electrons is negligible towards the overall enhancement in the AlNTs- $\mathrm{TiO}_{2}$ configuration. Furthermore, the contribution from plasmonic scattering was also studied by extracting the scattering and absorption spectra from the total extinction spectrum and carrying out measurements using an integrating sphere. Fig. 4E and $\mathrm{F}$ show the absorption and scattering of the AlNTs before and after combining with $\mathrm{TiO}_{2}$. It can clearly be seen that the scattering cross section is dominant for the AlNTs prior to $\mathrm{TiO}_{2}$ deposition; however, the absorption becomes dominant after embedding the AlNTs in $\mathrm{TiO}_{2}$. It seems that plasmonic near-field coupling is the dominating energy transfer channel rather than scattering, which correlates well with a recent report. ${ }^{40}$ Furthermore, we studied the thermal contribution during the photocurrent measurements at $365 \mathrm{~nm}$ by additionally irradiating the photoelectrodes simultaneously with a $\lambda=650 \mathrm{~nm}$ narrowband light source where aluminium absorbs light. The observed photocurrent remains stable and looks similar to Fig. 4A, thus revealing the negligible thermal contribution to the enhanced charge-carrier generation rate. The above 


\section{(A)}

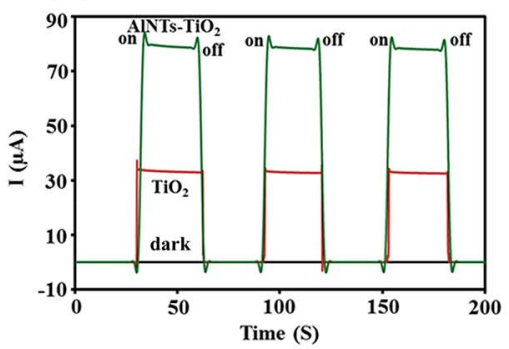

(C)

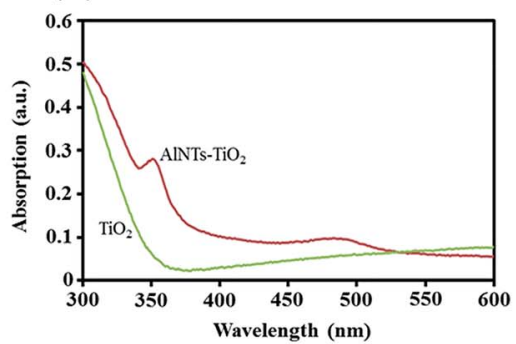

(E)

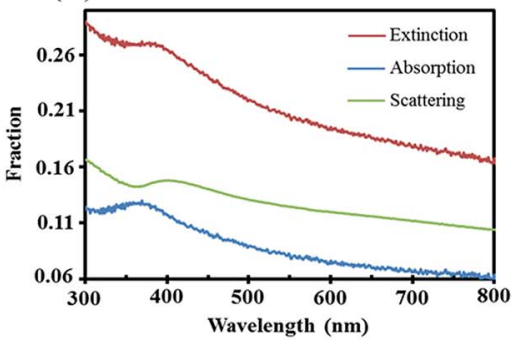

(B)

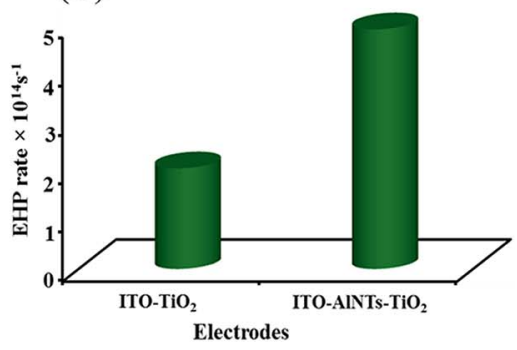

(D)

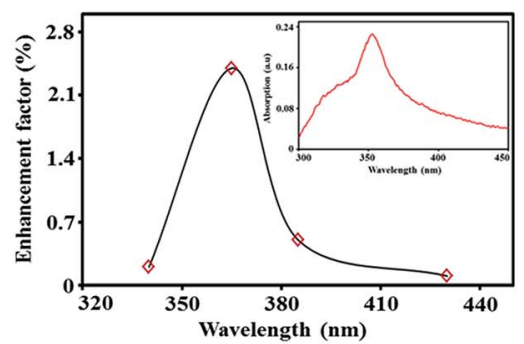

(F)

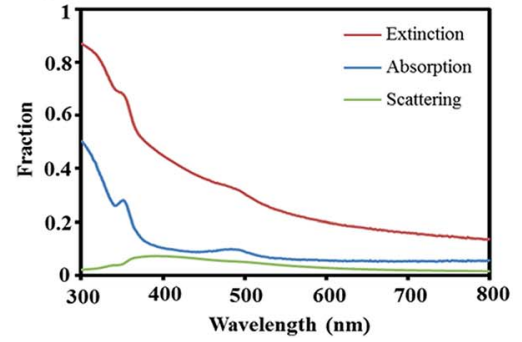

Fig. 4 (A) Photocurrent of ITO- $\mathrm{TiO}_{2}$ and ITO-AINTs- $\mathrm{TiO}_{2}$ in a $0.1 \mathrm{M} \mathrm{KNO}_{3}(\mathrm{pH} \sim 6)$ solution containing $10 \%$ ethanol, recorded under narrowband irradiation at $\lambda=365 \mathrm{~nm}(5$ $\mathrm{mW} \mathrm{cm}{ }^{-2}$ ) and the dark. (B) Charge-carrier generation rate of the $\mathrm{TiO}_{2}$ electrodes without and with AINTs. (C) Absorption spectra of $\mathrm{TiO}_{2}$ and $\mathrm{AINTs}-\mathrm{TiO}_{2}$. (D) Wavelength dependent photocurrent enhancement of the ITO-AINTs- $\mathrm{TiO}_{2}$ photoelectrode observed when using 340, 365, 385 and $430 \mathrm{~nm}$ narrowband light sources; the inset shows the absorption spectrum of the AINTs extracted from $4 C$. (E) Absorption and scattering fraction of the AlNTs. (F) Absorption and scattering fraction of AlNTs- $\mathrm{TiO}_{2}$.

characterizations strongly indicate a predominant contribution of the plasmonic near-field coupling to the enhancement of the charge carrier generation rate.

The stabilities of the fabricated electrodes were evaluated by measuring the photocurrent responses every month over a time period of 6 months. Fig. 5A shows the photocurrent responses of ITO-AlNTs exposed to the conditions used for the hot electron measurements described above. It can clearly be seen that the current response does not change significantly over the first month and decreases subsequently, thus indicating that the hot electron generation efficiency of the AlNTs is reduced with time. It is worth noting that even after 6 months the AlNTs demonstrate $80 \%$ of their initial efficiency, thus signifying that $\mathrm{Al}$ is more stable 
(A) 7.0

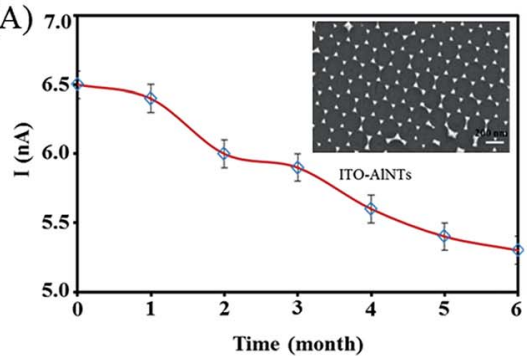

(B)

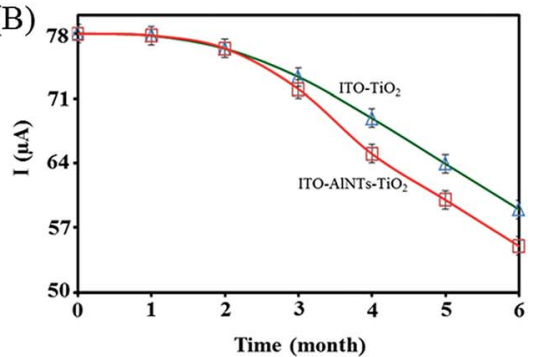

Fig. 5 Photocurrent responses of the (A) ITO-AINTs and (B) ITO-TiO 2 and ITO-AINTs$\mathrm{TiO}_{2}$ electrodes in a $0.1 \mathrm{M} \mathrm{KNO}_{3}(\mathrm{pH} \sim 6)$ solution containing $10 \%$ ethanol, recorded every month under narrowband irradiation at $\lambda=365 \mathrm{~nm}\left(5 \mathrm{~mW} \mathrm{~cm}{ }^{-2}\right)$. The inset in Fig. $5 \mathrm{~A}$ shows an SEM image of the AINTs recorded 6 months after fabrication.

than $\mathrm{Au}$ and $\mathrm{Ag}$. This is due to the fact that the self-limiting oxide (2-3 nm Al $\mathrm{AO}_{3}$ ) protects the structures from further oxidation. Furthermore, $\mathrm{Al}$ is insusceptible to surface diffusion, hence the shape of the structures remains, in contrast to Au and $\mathrm{Ag},{ }^{41}$ conserved. The inset of Fig. 5A shows an SEM image of the AlNTs taken 6 months after fabrication. Fig. 5B shows a stability study of the $\mathrm{ITO}^{-\mathrm{TiO}_{2}}$ and ITOAlNTs- $\mathrm{TiO}_{2}$ electrodes. The photocurrent responses of the $\mathrm{TiO}_{2}$ and $\mathrm{AlNTs}^{-\mathrm{TiO}_{2}}$ electrodes are reduced to $75 \%$ and $70 \%$, respectively, of the initial values.

\subsection{Photocatalytic ammonia production}

Plasmonic hot electron-driven nitrogen splitting was investigated and the results are shown in Fig. 6A. Under dark conditions, the control experiments revealed no significant ammonia production for the fused silica (FS) without AlNTs. Under illumination, the AlNTs deposited on the fused silica substrates catalyse the production of ammonia at a rate of $4 \times 10^{-5} \mu \mathrm{M} \mathrm{h}^{-1} \mathrm{~mW}^{-1} \mathrm{~cm}^{-2}$, which corresponds to a quantum efficiency of $2.5 \times 10^{-5} \%$. This clearly indicates that nitrogen splitting takes place at room temperature under atmospheric pressure. The observed ammonia production efficiency is comparable with the hot electron generation efficiency obtained from the electrochemical photocurrent measurements. Fig. 6B shows a schematic of the hot electron-driven charge injection into the nitrogen molecule. Hot electrons are generated from AlNTs as a result of nonradiative surface plasmon decay via Landau damping, ${ }^{42}$ and are distributed randomly with electron energies at $0 \mathrm{eV}$ to the excitation energy (365 $\mathrm{nm}$ ) above the Fermi level. Subsequently, hot electrons possessing a suitable energy are transferred into the lowest unoccupied molecular orbital (LUMO) of the adsorbed $\mathrm{N}_{2}$ molecule and break the $\mathrm{N}_{2}$ triple bond. The bond breaking mechanism is still not known. The hot electron generation and ammonia production efficiencies are modest, due to the very short lifetime of the hot electrons in the femtosecond range. The hot electrons rapidly lose their energy through electron-electron scattering followed by electron-phonon interactions. ${ }^{42}$ Furthermore, the absorption is less dominating in comparison with the scattering for $85 \mathrm{~nm}$ edge-length AlNTs, as inferred from Fig. 4E, thus suggesting that surface plasmon decay occurs mainly through a radiative process. 


\section{Faraday Discussions}

(A)

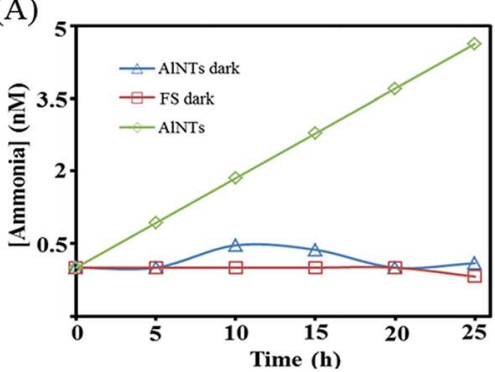

(C)

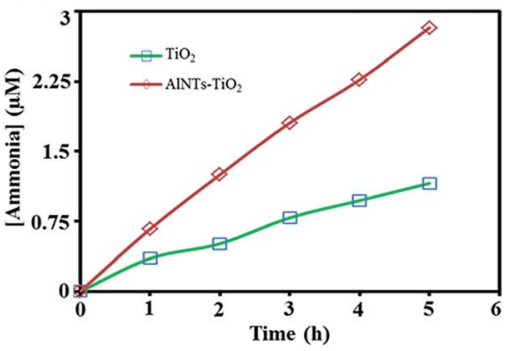

(E)

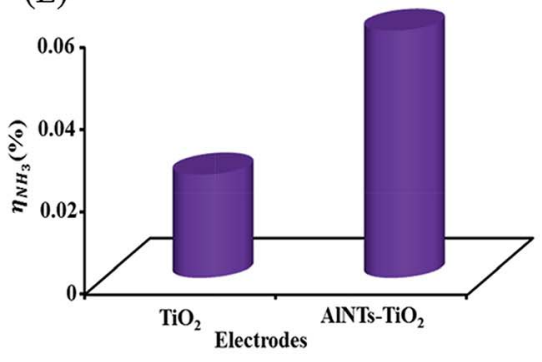

(B)

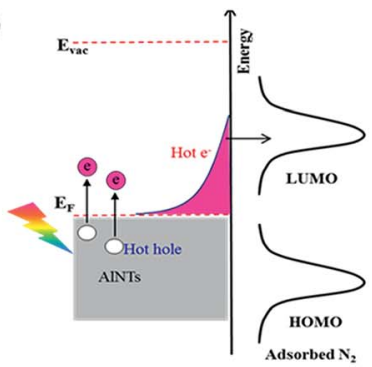

(D)

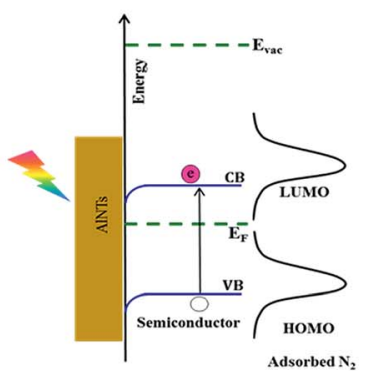

(F)

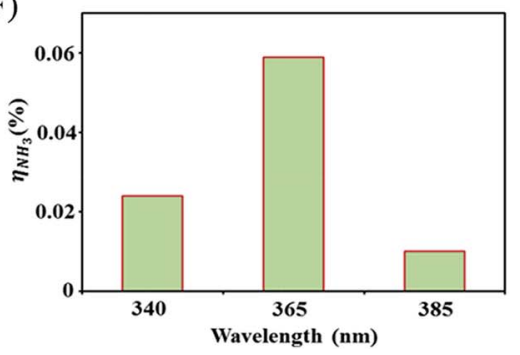

Fig. 6 (A) Plasmonic hot electron-driven ammonia production in a nitrogen-saturated solution $(\mathrm{pH} \sim 4$ ) containing $10 \%$ ethanol under dark conditions and illumination by a narrowband $365 \mathrm{~nm}$ beam with a $5 \mathrm{~mW} \mathrm{~cm}^{-2}$ power. (B) Schematic of hot electrondriven charge injection. (C) Plasmonic near-field coupling enhanced ammonia production in a nitrogen saturated solution $(\mathrm{pH} \sim 4$ ) containing $10 \%$ ethanol under illumination by a narrowband $365 \mathrm{~nm}$ beam with a $5 \mathrm{~mW} \mathrm{~cm}^{-2}$ power. (D) Schematic of plasmonic nearfield coupling enhanced charge injection. (E) Apparent quantum efficiencies of the $\mathrm{TiO}_{2}$ and AlNTs- $\mathrm{TiO}_{2}$ photoelectrodes. (F) Wavelength-dependent quantum efficiencies of the AlNTs $-\mathrm{TiO}_{2}$ photoelectrodes.

In a further step, the effect of near-field coupling on ammonia production was investigated. Fig. 6C shows the ammonia production on the bare $\mathrm{TiO}_{2}$ and AlNTs$\mathrm{TiO}_{2}$ photoelectrodes versus time.

Under dark conditions, both electrodes did not exhibit significant differences, whilst under illumination significant ammonia production was observed. It can be seen that ammonia production increases linearly with time, with the production rate estimated to be $0.04 \mu \mathrm{M} \mathrm{h}^{-1} \mathrm{~mW}^{-1} \mathrm{~cm}^{-2}$ for bare $\mathrm{TiO}_{2}$ and $0.1 \mu \mathrm{M}$ $\mathrm{h}^{-1} \mathrm{~mW}^{-1} \mathrm{~cm}^{-2}$ for AlNTs-TiO ${ }_{2}$. For the AlNTs-TiO ${ }_{2}$ sample the reaction rate was observed to be $\sim 2.5$ times higher than that for $\mathrm{TiO}_{2}$, which correlates well with the 
photocurrent measurements. Fig. 6D shows a schematic of the plasmonic nearfield coupling from the AlNTs to $\mathrm{TiO}_{2}$. EHP are short-living states leading to a short mean free path, on the order of $50 \mathrm{~nm}$, for the electrons. Hence, the confined plasmonic near-field with its short penetration depth into $\mathrm{TiO}_{2}$ favours the generation of EHP in the vicinity of the interface and reduces, therefore, the recombination probability. This shallowness has proved to be beneficial for nitrogen splitting. The apparent quantum efficiencies, $\eta$, of ammonia production for the $\mathrm{TiO}_{2}$ and $\mathrm{AlNTs}-\mathrm{TiO}_{2}$ photoelectrodes were calculated, with the results shown in Fig. 6E. The bare $\mathrm{TiO}_{2}$ and $\mathrm{AlNTs}^{-\mathrm{TiO}_{2}}$ substrates demonstrate, $\eta$ of $0.025 \%$ and $0.06 \%$, respectively. The observed $\eta$ is significantly higher than what has been previously reported..$^{\mathbf{3 4 3 - 4 5}}$ Furthermore, the highest $\eta$ was observed at the plasmonic resonance wavelength (Fig. 6F), revealing again the plasmonic nature of the process. In a control experiment, ammonia production without $\mathrm{N}_{2}$ replenishment was investigated. Fig. 7A clearly shows the stagnation of ammonia production after the dissolved $\mathrm{N}_{2}$ is exhausted.

A nitrogen splitting energy diagram is shown in Fig. 7B. The reduction of nitrogen on $\mathrm{TiO}_{2}$ is thermodynamically possible since the conduction band potential of $\mathrm{TiO}_{2}$ is at $-0.25 \mathrm{~V}$ with respect to the SHE at $\mathrm{pH} 0,{ }^{46}$ which is higher than the potential $(-0.092 \mathrm{~V})$ required for $\mathrm{N}_{2}$ splitting. In our experiments we used $\mathrm{pH} \sim 4$ to ensure the stability of the plasmonic photoelectrode and to provide enough protons for ammonia formation. Under these conditions the conduction band potential is $-0.486 \mathrm{~V}$ and the nitrogen splitting potential becomes $-0.328 \mathrm{~V}$ (according to the Nernst equation, i.e. $0.059 \times \mathrm{pH}$ ). Therefore, the energy balance allows for ammonia production under ambient conditions. The possible oxidation and reduction reactions taking place in the potential window are represented by the following equations:

$$
\begin{gathered}
h \nu \stackrel{\mathrm{TiO}_{2}}{\rightarrow} \mathrm{e}^{-}+\mathrm{h}^{+} \\
\mathrm{N}_{2}+6 \mathrm{H}^{+}+6 \mathrm{e}^{-} \rightarrow 2 \mathrm{NH}_{3}(-0.092 \mathrm{~V}) \\
\mathrm{C}_{2} \mathrm{H}_{5} \mathrm{OH}+2 \mathrm{~h}^{+} \rightarrow \mathrm{CH}_{3} \mathrm{CHO}+2 \mathrm{H}^{+}(0.249 \mathrm{~V}) \\
2 \mathrm{H}^{+}+2 \mathrm{e}^{-} \rightarrow \mathrm{H}_{2}(0 \mathrm{~V}) \\
4 \mathrm{OH}^{-}+4 \mathrm{~h}^{+} \rightarrow 2 \mathrm{H}_{2} \mathrm{O}+\mathrm{O}_{2}(1.23 \mathrm{~V})
\end{gathered}
$$

In addition to nitrogen reduction, the conduction band electrons can reduce protons and evolve molecular hydrogen gas (eqn (5)) since the required potential is well below the conduction band potential of $\mathrm{TiO}_{2}$. It is well known that $\mathrm{TiO}_{2}$ is a good photocatalyst not only for nitrogen reduction but also for hydrogen evolution, ${ }^{47}$ because both nitrogen and hydrogen adsorb well on the $\mathrm{TiO}_{2}$ surface. On the other hand, the holes generated in the valence band oxidize hydroxyl radicals leading to the production of water and the evolution of oxygen gas (eqn (6)). To compensate for the electrons used in nitrogen splitting, the electron donor ethanol refills the redundant holes in the VB of $\mathrm{TiO}_{2}$ (eqn (4)). 


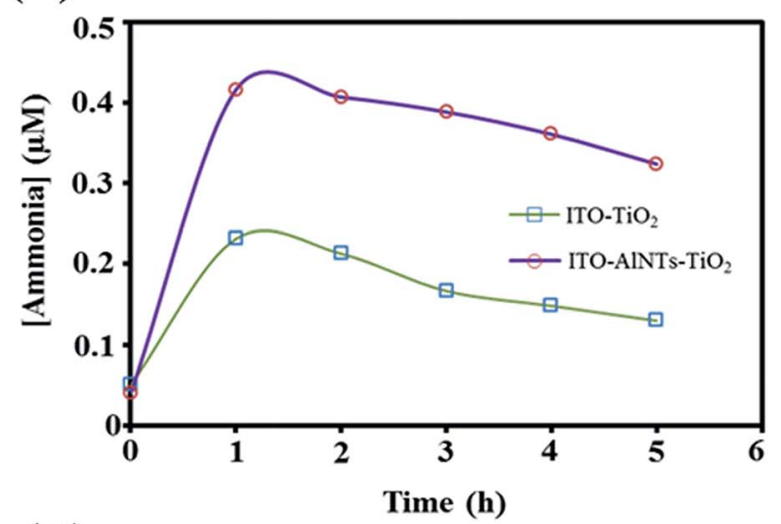

(B)

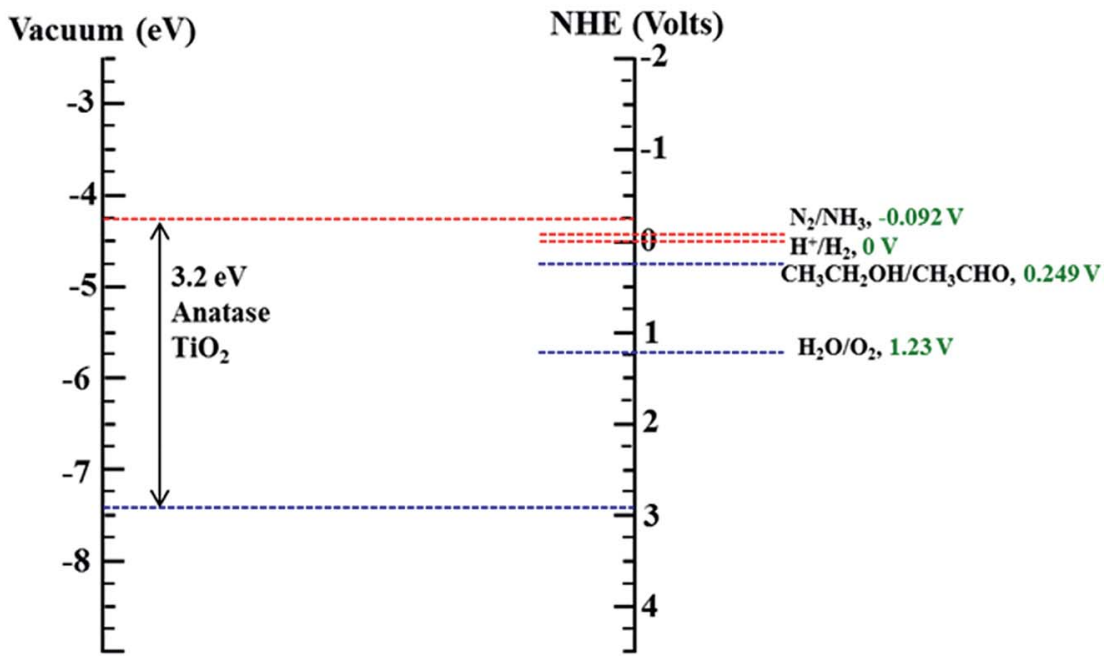

Fig. 7 (A) Ammonia production of irradiated $\mathrm{TiO}_{2}$ and $\mathrm{AINTs}-\mathrm{TiO}_{2}$ samples in the absence of a nitrogen gas flow through a nitrogen saturated solution $(\mathrm{pH} \sim 4)$ containing $10 \%$ ethanol under illumination by a narrowband $365 \mathrm{~nm}$ beam with a $5 \mathrm{~mW} \mathrm{~cm} \mathrm{~cm}^{-2}$ power. (B) Energy band alignment of anatase $\mathrm{TiO}_{2}$ and the standard oxidation and reduction potentials of the possible reactions at the band gap energy.

\section{Conclusion}

We have successfully demonstrated an alternative route for ammonia synthesis through nitrogen splitting at room temperature and under atmospheric pressure using the plasmonic effects of AlNTs. The samples were first characterised via SEM, XPS and XRD analysis, thus providing the band energies. The plasmonic hot electron generation from the AlNTs and near-field coupling to a semiconductor were studied in detail by carrying out electrochemical photocurrent measurements. The results revealed that the overall charge-carrier generation efficiency is dominated by a plasmonic near-field coupling effect, when illuminating $\mathrm{TiO}_{2}$ at a wavelength above the band gap energy. Hot electron-driven ammonia 
production was measured to be $4 \times 10^{-5} \mu \mathrm{M} \mathrm{h}^{-1} \mathrm{~mW}^{-1} \mathrm{~cm}^{-2}$ with a quantum efficiency of $2.5 \times 10^{-5} \%$. The AlNTs-TiO ${ }_{2}$ photoelectrode exhibits an ammonia production rate of $0.1 \mu \mathrm{M} \mathrm{h}^{-1} \mathrm{~mW}^{-1} \mathrm{~cm}^{-2}$, which is $\sim 2.5$ times higher than that of bare $\mathrm{TiO}_{2}\left(0.04 \mu \mathrm{M} \mathrm{h}^{-1} \mathrm{~mW}^{-1} \mathrm{~cm}^{-2}\right)$, thus indicating enhanced nitrogen splitting at room temperature and under atmospheric pressure. The overall conclusion is that at wavelengths above the band gap energy, the ammonia production rate can be enhanced by a near-field stemming from a plasmonic system, whereas hot electrons play a minor role in such a configuration. The present study offers a large-area AlNTs fabrication method using colloidal lithography, and provides a cheap, efficient and up-scalable method.

\section{Conflicts of interest}

There are no conflicts of interest.

\section{Acknowledgements}

This project was supported by the Gebert Ruf Stiftung, Switzerland (Grant no. GRS-039/16).

\section{References}

1 B. M. Hoffman, D. Lukoyanov, Z.-Y. Yang, D. R. Dean and L. C. Seefeldt, Chem. Rev., 2014, 114, 4041-4062.

2 S. Ritter, Chem. Eng. News, 2008, 86, 43.

3 A. R. Jawahery, Energy Efficiency and $\mathrm{CO}_{2}$ Emissions in Ammonia Production, International Fertilizer Industry Association, Paris, France, 2009.

4 S. Giddey, S. P. S. Badwal and A. Kulkarni, Int. J. Hydrogen Energy, 2013, 38, 14576-14594.

5 S. Licht, B. Cui, B. Wang, F.-F. Li, J. Lau and S. Liu, Science, 2014, 345, 637-640.

6 J. A. Pool, E. Lobkovsky and P. J. Chirik, Nature, 2004, 427, 527-530.

7 K. Arashiba, Y. Miyake and Y. Nishibayashi, Nat. Chem., 2011, 3, 120-125.

8 R. I. Bickley and V. Vishwanathan, Nature, 1979, 280, 306-308.

9 O. Rusina, A. Eremenko, G. Frank, H. P. Strunk and H. Kisch, Angew. Chem., Int. Ed., 2001, 40, 3993-3995.

10 G. N. Schrauzer and T. D. Guth, J. Am. Chem. Soc., 1977, 99, 7189-7193.

11 A. Fujishima and K. Honda, Nature, 1972, 238, 37-38.

12 K. T. Ranjit, T. K. Varadarajan and B. Viswanathan, J. Photochem. Photobiol., A, 1996, 96, 181-185.

13 M. M. Taqui Khan and N. Nageswara Rao, J. Photochem. Photobiol., A, 1991, 56, 101-111.

14 M. M. Taqui Khan, R. C. Bhardwaj, C. Bhardwaj and N. Nageswara Rao, J. Photochem. Photobiol., A, 1992, 68, 137-146.

15 M. M. Taqui Khan, D. Chatterjee and M. Bala, J. Photochem. Photobiol., A, 1992, 67, 349-352.

16 X.-C. Ma, Y. Dai, L. Yu and B.-B. Huang, Light: Sci. Appl., 2016, 5, e16017.

17 S. Linic, U. Aslam, C. Boerigter and M. Morabito, Nat. Mater., 2015, 14, 567576. 
18 M. W. Knight, Y. Wang, A. S. Urban, A. Sobhani, B. Y. Zheng, P. Nordlander and N. J. Halas, Nano Lett., 2013, 13, 1687-1692.

19 S. C. Warren and E. Thimsen, Energy Environ. Sci., 2012, 5, 5133-5146.

20 S. Mukherjee, L. Zhou, A. M. Goodman, N. Large, C. Ayala-Orozco, Y. Zhang, P. Nordlander and N. J. Halas, J. Am. Chem. Soc., 2014, 136, 64-67.

21 S. Mukherjee, F. Libisch, N. Large, O. Neumann, L. V. Brown, J. Cheng, J. B. Lassiter, E. A. Carter, P. Nordlander and N. J. Halas, Nano Lett., 2013, 13, 240-247.

22 S. Feng, M. Wang, Y. Zhou, P. Li, W. Tu and Z. Zou, APL Mater., 2015, 3, 104416.

23 K. Li, N. J. Hogan, M. J. Kale, N. J. Halas, P. Nordlander and P. Christopher, Nano Lett., 2017, 17, 3710-3717.

24 F. Chen, Q. Yang, C. Niu, X. Li, C. Zhang and G. Zeng, RSC Adv., 2015, 5, 6315263164.

25 L.-B. Zhao, X.-X. Liu and D.-Y. Wu, J. Phys. Chem. C, 2016, 120, 1570-1579.

26 B. Wu, J. Lee, S. Mubeen, Y.-S. Jun, G. D. Stucky and M. Moskovits, Adv. Opt. Mater., 2016, 4, 1041-1046.

27 T. Oshikiri, K. Ueno and H. Misawa, Angew. Chem., Int. Ed., 2014, 53, 98029805.

28 T. Oshikiri, K. Ueno and H. Misawa, Angew. Chem., Int. Ed., 2016, 55, 39423946.

29 K. Thyagarajan, C. Santschi, P. Langlet and O. J. F. Martin, Adv. Opt. Mater., 2016, 4, 871-876.

30 B. Gallinet, A. M. Kern and O. J. F. Martin, J. Opt. Soc. Am. A, 2010, 27, 22612271.

31 T. A. F. König, P. A. Ledin, J. Kerszulis, M. A. Mahmoud, M. A. El-Sayed, J. R. Reynolds and V. V. Tsukruk, ACS Nano, 2014, 8, 6182-6192.

32 T. Siefke, S. Kroker, K. Pfeiffer, O. Puffky, K. Dietrich, D. Franta, I. Ohlídal, A. Szeghalmi, E.-B. Kley and A. Tünnermann, Adv. Opt. Mater., 2016, 4, 1780-1786.

33 A. D. Rakić, Appl. Opt., 1995, 34, 4755-4767.

34 T. Oshikiri, K. Ueno and H. Misawa, Angew. Chem., Int. Ed., 2014, 53, 98029805.

35 D. O. Scanlon, C. W. Dunnill, J. Buckeridge, S. A. Shevlin, A. J. Logsdail, S. M. Woodley, C. R. A. Catlow, M. J. Powell, R. G. Palgrave, I. P. Parkin, G. W. Watson, T. W. Keal, P. Sherwood, A. Walsh and A. A. Sokol, Nat. Mater., 2013, 12, 798-801.

36 R. Beranek, Adv. Phys. Chem., 2011, 1-20.

37 J. Schneider, M. Matsuoka, M. Takeuchi, J. Zhang, Y. Horiuchi, M. Anpo and D. W. Bahnemann, Chem. Rev., 2014, 114, 9919-9986.

38 X. Shi, K. Ueno, N. Takabayashi and H. Misawa, J. Phys. Chem. C, 2013, 117, 2494-2499.

39 P. Christopher, H. Xin, A. Marimuthu and S. Linic, Nat. Mater., 2012, 11, 10441050.

40 U. Aslam, S. Chavez and S. Linic, Nat. Nanotechnol., 2017, 12, 1000-1005.

41 X. Wang, C. Santschi and O. J. F. Martin, Small, 2017, 13, 1700044.

42 K. Wu, J. Chen, J. R. McBride and T. Lian, Science, 2015, 349, 632-635.

43 M. Ali, F. Zhou, K. Chen, C. Kotzur, C. Xiao, L. Bourgeois, X. Zhang and D. R. MacFarlane, Nat. Commun., 2016, 7, 1-5. 
44 T. Oshikiri, K. Ueno and H. Misawa, Angew. Chem., Int. Ed., 2016, 55, 39423946.

45 H. Zeng, S. Terazono and T. Tanuma, Catal. Commun., 2015, 59, 40-44.

46 M. Grätzel, Nature, 2001, 414, 338.

47 D. B. Ingram, P. Christopher, J. L. Bauer and S. Linic, ACS Catal., 2011, 1, 14411447. 